\title{
Medición de las condiciones habita es: una propuesta metodológica para analizar desigualdades inter e intraterritorios urbanos ${ }^{*}$
}

\begin{tabular}{|l|l|}
\hline Fecha de recepción: 15 de junio de 2016 & Fecha de aceptación: 19 de enero de $2017 \quad$ Disponible en línea: 21 de agosto de 2017 \\
Eugenio Actis Di Pasquale & \\
\hline Doctor con mención en Ciencias Sociales y Humanas (UNQ) & Profesor adjunto en la FCEyS-UNMdP de Introducción \\
\hline a la Economía, Política Económica I y Mercado de Trabajo y Relaciones Laborales & edipasq@mdp.edu.ar \\
\hline Director del Grupo de estudios del trabajo FCEyS-UNMdP & eugenioactis@yahoo.com.ar \\
\hline
\end{tabular}

Resumen

Palabras clave

La medición de las condiciones habitacionales de un territorio resulta de especial interés para la implementación de políticas públicas. Los métodos tradicionales se concentran en la medición de los déficits y dejan de lado aquella parte de la población que se encuentra cerca del umbral. Con base en propuestas específicas, estándares de organismos internacionales, legislación nacional y la importante participación de especialistas, en este estudio se genera una metodología cuantitativa, que evita esa pérdida de información al incluir a toda la población de un territorio, asignándole un puntaje a cada persona de acuerdo con las características del hogar que habita. Para validar la propuesta, se presenta una aplicación con datos de Argentina, donde se constata su versatilidad, dado que permite detectar problemáticas particulares en cada dimensión, y también consigue analizar el nivel promedio, la distribución intra e interterritorios y la pérdida de bienestar por desigualdad.

bienestar social; distribución del ingreso; indicadores de desarrollo; vivienda

\footnotetext{
Artículo de investigación científica, producto de una línea de trabajo que se inicia con la tesis doctoral: Bienestar social, resignificación del concepto y de su operacionalización. Un aporte metodológico aplicado al caso argentino, finalizada en julio de 2013 y dirigida por el doctor Javier Balsa. Continuada por el proyecto de investigación De la teoría económica del bienestar pasando por el buen vivir hasta la operacionalización de la felicidad. Debates teóricos y construcción de indicadores, su aplicación en Argentina 1994-2014 y su testeo a nivel local, que inició en marzo de 2015. Proyecto financiado por la Universidad Nacional de Mar del Plata (código 15/D121) y dirigido por la doctora María Estela Lanari.
}

Cómo citar este artículo: Actis Di Pasquale, E. (2017). Medición de las condiciones habitacionales: una propuesta metodológica para analizar desigualdades inter e intraterritorios urbanos. Cuadernos de Vivienda y Urbanismo, 10(20), 46-67. https://doi.org/10.11144/Javeriana.cvu10-20.mchp 


\section{Measuring Housing Conditions:}

a Methodological Proposal to Analyze Inequalities in the Inter and Intraurban Territories

Abstract The measurement of the housing conditions of a territory is particularly important for the implementation of public policies. Traditional methods focus on the measurement of deficits and leave aside that part of the population which is closer to the threshold. Based on specific proposals, the standards of international organizations, national legislation, and a significant participation of specialists, this study proposes a quantitative methodology, which avoids said loss of information by including the entire population of a territory, assigning a score to each person according to the characteristics of the household they live in. To validate this proposal, we present an application with data from Argentina, where its versatility is verified given that it allows for the detection of issues in each dimension while also getting to analyze the average level, the intra and inter-territorial distribution, and the welfare loss due to inequality.

Keywords social welfare; income distribution; development indicators; housing

\section{Aferição das condições habitacionais:}

proposta metodológica para analisar desigualdades inter e intraterritórios urbanos

Resumo A aferição das condições habitacionais de um território resulta de especial interesse para a implementação de política pública. Os métodos tradicionais concentram-se na mediçáo dos déficits e deixam de lado aquela parte da população que se encontra perto do umbral. Com base em propostas específicas, padrões de organismos internacionais, legislação nacional e a importante participação de especialistas, neste estudo gera-se uma metodologia quantitativa, que evita tal perda de informação ao incluir a população inteira de um território, outorgando-lhe pontuação a cada pessoa de acordo às caraterísticas da moradia que habita. Para validar a proposta, apresenta-se aplicação com dados da Argentina, onde constata-se a versatilidade, dado que permite detectar problemáticas particulares em cada dimensão e, mesmo, consegue analisar o nível médio, a distribuição intra e interterritórios e a perda de bem-estar por desigualdade. 


\section{Introducción}

Las condiciones habitacionales de la población representan uno de los componentes básicos en los estudios sobre bienestar social, calidad de vida y pobreza. Habitar una vivienda digna nos asegura protección frente a inclemencias del tiempo, un ambiente inmediato sano y un espacio de intimidad y privacidad. Por ende, es una precondición material para que, siguiendo la terminología de Amartya Sen, toda persona pueda ser y hacer lo que tiene razones para valorar (Sen, 2000).

En América Latina y el Caribe el acceso a la vivienda adecuada es desigual, siendo esta disparidad multidimensional, dado que no solo se refiere a las condiciones materiales de techo y piso, sino también al acceso a un saneamiento adecuado y de seguridad jurídica en la tenencia. En este sentido, la mayor parte de los estudios específicos que se dedican a cuantificar la problemática habitacional lo hacen analizando dos tipos de carencias: las necesidades o requerimientos de vivienda que tienen las unidades domésticas — déficit cuantitativo- y las viviendas en condiciones de atender a satisfacción las necesidades habitacionales —déficit cualitativo- (Rodríguez, 1999; Szalachman, 2000; Cecchini, 2005).

Dadas las múltiples dimensiones que componen este tipo de estudios, para lograr construir un índice sintético que dé cuenta del dominio específico del contenido de lo que se mide, en general se procede por medio de las siguientes etapas: 1) se seleccionan y definen las dimensiones que la integran; 2) se fijan umbrales que delimitan lo adecuado de lo inadecuado en cada una de esas dimensiones; 3) se construyen los índices parciales para cada dimensión, que por lo general son indicadores agregados que dan cuenta solo del porcentaje de población que habita en viviendas que requieren atención inmediata; y al final, en algunos estudios 4) se elabora una medida sintética.

Sin embargo, este procedimiento no es tarea sencilla dado que en cada una de esas etapas nos enfrentamos a una serie de problemas y limitaciones metodológicas. En primer lugar, la multidimensionalidad del concepto nos fuerza a elegir solo las variables más significativas para definir la amplitud del concepto en lo operativo. De no hacerlo en forma correcta se producirá una falta de correspondencia entre el concepto y el indicador, es decir, estaríamos perdiendo validez interna. En segundo lugar, en la etapa de definición de los umbrales, al fijar límites de acuerdo con algún criterio preestablecido, se dejan por fuera del indicador —y por ende del análisis — a aquellas situaciones que están muy próximas a ese umbral. Sin embargo, ese grupo poblacional presenta cierto grado de vulnerabilidad y podría caer en un déficit habitacional frente a algún acontecimiento inesperado. En tercer lugar y, seguido de lo anterior, los indicadores elaborados a partir de un criterio de déficit no son válidos para conocer la distribución, sino tan solo las situaciones inadecuadas. Por último, la elaboración de un índice sintético trae consigo una diversidad de problemáticas en sí mismas, que incluyen conseguir una estandarización adecuada de los índices parciales, utilizar una ponderación que refleje la presencia de déficits en una o más dimensiones y agregar toda la información en una medida única que refleje las características de los hogares. 
En virtud de ello, este trabajo tiene como objetivo elaborar un índice sintético — en una escala numérica de cero a uno- que refleje el nivel de las condiciones habitacionales que posee cada uno de los hogares. De esta manera, al evaluar a toda la población evitamos la pérdida de información estadística de los casos próximos a los umbrales. Esto genera indicadores agregados para realizar múltiples análisis sobre la desigualdad de la distribución inter e intraterritorios, lo que permite detectar problemáticas habitacionales a partir de un baremo mucho más amplio.

El presente trabajo está organizado en cuatro apartados. En el siguiente realizamos una definición conceptual de las condiciones habitacionales. Luego, presentamos la propuesta metodológica cuantitativa que permite transformar variables de distinta naturaleza — categóricas o cuantitativas - en índices equiparables que representan niveles de condiciones habitacionales. En seguida se exponen las particularidades en la elaboración de cada índice. Al final, realizamos un ejercicio de validación empírica con datos del IV trimestre de 2014 de la EPH-Indec de Argentina.

\section{Una definición conceptual de las condiciones habitacionales}

En la literatura no hay consenso sobre lo que se considera una vivienda, debido a que los elementos que la constituyen difieren de acuerdo con la cultura y a las condiciones ambientales propias del entorno geográfico. Sin embargo, se ha avanzado en distinguir algunas características elementales, según las funciones que se le atribuyen y que permiten caracterizar un alojamiento adecuado (Doyal y Gough, 1994; Rodríguez, 1999; Szalachman, 2000; García-Huidobro, 2005; Cecchini, 2005). A grandes rasgos podemos senalar las siguientes:
1. Protección frente a las inclemencias del medio - lluvia, calor, frío y humedad-. Resulta fundamental el estado de la vivienda $y$ de la calidad de sus materiales, en especial el techo, las paredes y el piso.

2. Ofrecimiento de un ambiente inmediato sano. Depende en gran medida de la disponibilidad de un conjunto mínimo de servicios básicos en la vivienda, tales como el abastecimiento de agua potable, disponibilidad de electricidad y un sistema adecuado para la eliminación de excretas (Szalachman, 2000).

3. Disponibilidad de un espacio de intimidad y privacidad. El requisito es la existencia de una superficie mínima para cada individuo, lo cual nos remite a la noción de hacinamiento, que es un concepto relativo que varía según el nivel de desarrollo de las sociedades, el momento histórico y las particularidades culturales. Para aproximar a esta idea, por lo general se utilizan tres criterios diferenciados, que dependerán del juicio normativo sobre niveles apropiados de ocupación, densidad y privacidad (Spicker, Álvarez y Gordon, 2009). Sin embargo, en América Latina, ningún censo de población y vivienda releva información sobre la superficie (Rodríguez, 1999). En este sentido, si bien las dos formas indicativas de esta problemática son la cantidad de hogares por vivienda - hacinamiento por hogar o cohabitación-y la relación de personas por cuarto - hacinamiento por cuarto-, optamos por esta última debido a cuestiones metodológicas y empíricas que se detallan con más atención en la Sección 4 (Lentini y Palero, 1997).

4. Una condición adicional que se incorpora en diferentes estudios es la seguridad jurídica de tenencia (ONU-Hábitat, 2003; Salvia, 2004). En el Código Civil y Comercial de la República Argentina (CCC) es entendida como la protección contra el desalojo forzado, característica que tiene una íntima relación con la 
posesión jurídica de la vivienda por parte del hogar (Ramírez, 2002).

Estos cuatro elementos representan las dimensiones seleccionadas para la medición de las condiciones habitacionales. A continuación, enunciamos los índices que las componen y las etapas implicadas en su elaboración.

\section{Propuesta general de medición de las condiciones habitacionales}

De acuerdo con los antecedentes específicos, estándares de organismos internacionales, legislación nacional y, muy importante, la participación de especialistas, elaboramos estos cinco índices parciales que conforman el índice sintético de las condiciones habitacionales (Tabla 1).

\section{Etapas de elaboración}

Cada uno de estos es el resultado de un proceso que consta de las siguientes etapas generales: 1. conceptualización general de los valores

Tabla 1

Índice de condiciones habitacionales de referencia; 2. fijación de valores de referencia a cada indicador; 3. cálculo de los valores intermedios en los intervalos; 4. elaboración del índice sintético para cada persona, y 5. cálculo de medidas de distribución.

En la primera etapa se elabora una escala conceptual de conversión con límites que oscilan entre 0 y 1 . Los valores de referencia sugeridos son: 0 (cero) que implica la peor situación; 0,40 que representa un nivel bajo y que delimita la situación considerada como déficit; 0,70 que indica un nivel medio, tal vez calificable como una situación aceptable; y 1 constituye un nivel alto, donde pueden convivir una diversidad de situaciones adecuadas.

La segunda etapa es la asimilación de un determinado valor a una variable - o indicador — sin estandarizar a un valor de referencia, se realiza por medio de un proceso que incluye: la revisión de estudios previos específicos a cada componente, el análisis de estándares y recomendaciones de los organismos internacionales, la exploración de la legislación nacional (en los índices que corresponda) y, de gran importancia, la participación

\begin{tabular}{|c|c|c|c|c|}
\hline Índice sintético & Dimensiones & Índices parciales & $\begin{array}{l}\text { Naturaleza de las } \\
\text { variables de origen }\end{array}$ & $\begin{array}{l}\text { Cálculo de valores } \\
\text { intermedios }\end{array}$ \\
\hline \multirow{4}{*}{ 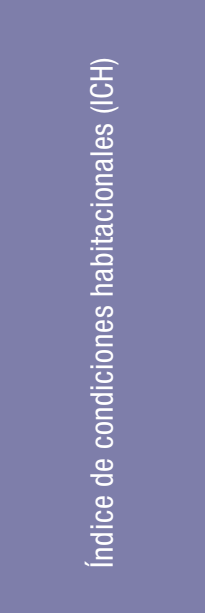 } & $\begin{array}{l}\text { Protección frente a las } \\
\text { inclemencias del medio }\end{array}$ & $\begin{array}{l}\text { 1- Índice de techo-piso } \\
\text { (ITP) }\end{array}$ & $\begin{array}{l}\text { Combinación de variables } \\
\text { categóricas ordinales }\end{array}$ & \multirow[b]{2}{*}{ Puntos fijos combinados } \\
\hline & $\begin{array}{c}\text { Ofrecimiento de un } \\
\text { ambiente inmediato sano }\end{array}$ & $\begin{array}{c}\text { 2- Índice de acceso al } \\
\text { agua (IAA) } \\
\text { 3- Índice sobre } \\
\text { disposición de excretas } \\
\text { y saneamiento (IDE) }\end{array}$ & $\begin{array}{c}\text { Combinación de variables } \\
\text { categóricas ordinales y } \\
\text { nominales }\end{array}$ & \\
\hline & $\begin{array}{l}\text { Disponibilidad de un } \\
\text { espacio de intimidad y } \\
\text { privacidad }\end{array}$ & $\begin{array}{l}\text { 4- Índice de personas por } \\
\text { dormitorio (IPD) }\end{array}$ & $\begin{array}{l}\text { Variable cuantitativa } \\
\text { discreta }\end{array}$ & LST por tramos \\
\hline & $\begin{array}{c}\text { Seguridad jurídica de } \\
\text { tenencia }\end{array}$ & $\begin{array}{l}\text { 5- Índice sobre seguridad } \\
\text { jurídica en la tenencia } \\
\text { (ISJT) }\end{array}$ & Variable categórica ordinal & Puntos fijos \\
\hline
\end{tabular}


de especialistas o informantes calificados. De esta manera, se busca un consenso intersubjetivo para obtener una medida válida desde el punto de vista de las voces calificadas (Bostwick y Kyte, 2005).

La tercera etapa se corresponde con la estimación de los valores intermedios a los de referencia, momento en que se vuelve a entrevistar a los especialistas con el objetivo de mantener la validez externa de las medidas. La técnica empleada para determinar los valores intermedios es diferente de acuerdo con la naturaleza de la variable de origen (Tabla 1). En el caso de las variables categóricas, el puntaje de cada categoría se determina de la misma forma que los de referencia; por ende, lo denominamos puntos fijos. En los índices que surgen de una combinación de variables categóricas, se calculó el producto cruzado de esos valores y lo nombramos puntos fijos combinados. En cambio, en el índice que proviene de variables cuantitativas, los valores intermedios se calculan mediante el uso de una modificación del método de Linear Scaling Technique (LST) propuesto por Drewnowski y Scott, (1966), que denominamos LST por tramos, dado que la transformación se realiza entre valores de correspondencia intermedios y no entre los extremos.

La fórmula utilizada difiere según estemos en presencia de indicadores positivos o negativos. En términos generales, para los primeros, la fórmula utilizada para cada tramo de referencia es:

$$
I_{\text {interm }}=\frac{X_{i}-\operatorname{MIN}_{j}}{M A X_{j}-M I N_{j}} \times \text { rango }_{j}+\min _{j}
$$

Y para los indicadores negativos:

$$
I_{\text {interm }}=\frac{M A X_{j}-X_{i}}{M A X_{j}-M I N_{j}} \times \text { rango }_{j}+\min _{j}
$$

Donde:

, $\mathrm{I}_{\text {interm }}$ : es el valor intermedio del índice parcial.

, $\mathrm{X}_{\mathrm{i}}$ : es el valor del indicador sin estandarizar del territorio geográfico i.

> MAX: límite superior del tramo $\mathrm{j}$ de ese indicador sin estandarizar.

- MIN $\mathrm{j}_{\mathrm{j}}$ límite inferior del tramo $\mathrm{j}$ de ese indicador sin estandarizar.

, rango ${ }_{j}$ es la diferencia entre max y min del tramo $\mathrm{j}$ de valores de referencia.

min: límite inferior del tramo $\mathrm{j}$ de los valores de referencia.

max: límite superior del tramo j de los valores de referencia.

La técnica propuesta logra superar las limitaciones del LST estándar, dado que se obtienen medidas equiparables que no solo posibilitan la interpretación de los niveles acordados por consenso intersubjetivo, sino que se encuentran en condiciones de ser agregados por algún método particular que no elimine esas propiedades.

Por último, en la cuarta etapa, el cálculo de los índices parciales lo efectuamos en orden de las personas - y no del aglomerado - que habitan una misma unidad habitacional. Por ende, el índice de condiciones habitacionales (ICH) también se calcula para cada persona. Este cálculo lo efectuamos con lo que denominamos ponderación de la media geométrica g, que se diferencia de la media geométrica ponderada en que esta última otorga los pesos en el cálculo, mientras que la primera lo hace luego de efectuado el mismo. De esta manera, logramos obtener un índice que no solo presupone que todos los indicadores deben ser cercanos al valor más alto — siempre refiriéndonos al mismo hogar-, sino también que 
ninguno de ellos sea inferior a los valores de referencia que fijamos ${ }^{1}$.

El procedimiento toma en cuenta las siguientes condiciones: 1) Si el hogar presenta al menos un índice parcial con valor menor a 0,40 entendemos que la situación no es aceptable y, por ende, el índice sintético debería ser menor a 0,70. De esta manera, cuando hay un índice parcial con estas características, multiplicamos g por 0,70 ; si hay dos, por 0,80; tres, 0,90 ; cuatro y cinco, 0,95 . Las diferentes ponderaciones se justifican en una propiedad matemática en la que, a medida que hay más valores por debajo de 0,40 , g converge a dichos valores. Por lo tanto, al utilizar diferentes ponderaciones se evita que el índice sintético sea menor que el más bajo de los parciales; 2) si el hogar tiene índices con valores entre 0,40 y 0,70 , se considera que las condiciones habitacionales deben estar distanciadas de lo adecuado - es decir, de $1-y$, por lo tanto, se multiplica el resultado de la media geométrica por 0,95 ; 3) si el hogar no tiene índices con valores menores o iguales a 0,70 , el resultado es la media geométrica.

La quinta etapa se lleva a cabo una vez estimados los índices parciales y sintéticos individuales, en la cual, la propuesta de medición permite efectuar una variedad de análisis distributivos intra e interaglomerados. De esta manera, se consigue una visión integral tanto de cada dimensión como también del conjunto de las condiciones habitacionales. En definitiva para evaluar las condiciones habitacionales de las personas, podemos realizar cuatro exámenes:

1. Media Aritmética (I-Med). Es el promedio de puntaje de cada aglomerado calculado a partir del puntaje de cada persona que habita en un hogar determinado. Se calcula para cada índice: ITP-Med, IAA-Med, IDE-Med, IPDMed, ISJT-Med y ICH-Med.

2. Análisis percentílico. Permite estudiar y comparar tanto la distribución personal en un mismo territorio geográfico, como también los distintos niveles que alcanza un mismo estrato poblacional entre los territorios geográficos. En este sentido, el estudio distributivo brinda una primera aproximación sobre las diferencias de bienestar intra e interterritorios. Asimismo, conociendo la interpretación conceptual de cada valor que adopta el indicador, se puede detectar el porcentaje de población que se encuentra por encima o por debajo de un determinado nivel.

3. Índice ajustado por desigualdad (I-Des). Surge del producto entre cada I-Med y un factor corrector que calculamos como 1 menos la varianza $^{2}$. Por ende, se obtienen otros seis índices de los aglomerados: ITP-Des, IAA-Des, IDE-Des, IPD-Des, ISJT-Des y ICH-Des.

$$
I-D e s=I M e d \times\left(1-\sigma^{2}\right)
$$

Cabe destacar que si bien $\sigma^{2}$ posee limitaciones como indicador de desigualdad, tales como la dependencia de escala, en esta propuesta no tienen ninguna implicación, dado que la escala que adoptamos siempre es entre 0 y 1 . En correspondencia con PNUD (2010), el I-Med es un índice del bienestar potencial, dado que da cuenta del nivel de bienestar que puede obtenerse si no existiera desigualdad en la distribución. Mientras que el I-Des representa el nivel de bienestar real, ya que toma en cuenta la desigualdad.

4. Medición de la pérdida de bienestar por desigualdad. Una vez obtenidos los I-Med e I-Des, el cálculo de la diferencia porcentual 
entre ambos mide la pérdida de bienestar potencial debido a la desigualdad:

$$
\text { Pérdida }=1-\frac{I-D e s}{I-M e d} \times 100
$$

A continuación detallamos los aspectos específicos de la elaboración de cada uno de los cinco índices, en particular los correspondientes a las etapas 2 y 3 , teniendo en cuenta las limitaciones de la fuente de datos a utilizar, es decir, la EPH-Indec.

\section{Particularidades de la elaboración de cada índice parcial}

\section{1. Índice de calidad del techo-piso (ITP)}

Está basado en propuestas cuantitativas (Madariaga, 2003), como también aquellas que combinan la información de dos o más variables categóricas ordinales en una sola de la misma naturaleza (Indec, 2003; Szalachman, 2000) ${ }^{3}$. De esta manera se elaboró un índice que combina solo la información sobre las características del techo y piso, dado que la EPH-Indec no releva datos sobre la calidad de las paredes.

El puntaje asignado al techo se estableció con base en criterios de durabilidad, resistencia, aislamiento térmico e hidrófugo (IRAM, 1996), agregando como elemento adicional el cielorraso, que se utiliza para diferenciar a los techos de chapa de metal de los de fibrocemento. De esta manera, se tiene los siguientes tipos de techo: 1. membrana/ cubierta asfáltica; 2 . baldosa/losa sin cubierta; 3. pizarra/teja; 4. chapa de metal sin cubierta; 5. chapa de fibrocemento/plástico; 6. chapa de cartón; 7. cańa/tabla/paja con barro/paja sola, y 8. departamento en propiedad horizontal. Asimismo, se reagruparon en las siguientes categorías según la existencia o no de cielorraso: piso tipo A opciones 1 a 5 y 8 con cielorraso; piso tipo
B opciones 1, 2 y 3 y 8 sin cielorraso; piso tipo $\mathrm{C}$ opciones 4 y 5 sin cielorraso y piso tipo D las opciones 6 y 7 con o sin cielorraso.

El puntaje asignado al piso está relacionado con la higiene, prevención de enfermedades y la presencia de elementos resistentes, aislante hidrófugo y terminación: piso tipo A en mosaico/ baldosa/madera/cerámica/alfombra; piso tipo B en cemento/ladrillo fijo, y piso tipo $\mathrm{C}$ en ladrillo suelto/tierra. A partir del producto de ambos puntajes, de techo y piso, surge el ITP (Tabla 2).

Tabla 2

Índice de calidad del techo-piso (ITP)

\begin{tabular}{cccccc}
\multirow{2}{*}{ Piso } & \multicolumn{5}{c}{ Techo } \\
\cline { 2 - 5 } & A & B & C & D \\
\hline A & 1.00 & 0.90 & 0.60 & 0.30 \\
\hline B & 0.70 & 0.63 & 0.42 & 0.21 \\
\hline C & 0.30 & 0.27 & 0.18 & 0.09 \\
\hline
\end{tabular}

Fuente: elaboración propia

La diferencia entre los pisos tipos A y B es mayor que entre los techos tipo A y B debido a que, en los primeros, el cielorraso mejora el aislamiento térmico ya existente; en cambio, el piso de cemento o ladrillo fijo remite a pisos incompletos, sin terminación, o en el caso del ladrillo una terminación no óptima sobre todo en el aspecto de la higiene. El techo tipo C no llega a ser aceptable debido al mal aislamiento térmico que genera. Por último, el techo tipo $\mathrm{D}$ y el piso tipo $\mathrm{C}$ representan déficits en los parámetros considerados como relevantes, por lo tanto, no llegan a satisfacer condiciones básicas o mínimas.

\section{2. Índice de acceso al agua (IAA)}

El criterio utilizado en su elaboración es el que tiene mayor aceptación en la literatura y da cuenta de la disponibilidad del servicio de agua sin esfuerzo físico ni pérdida de tiempo excesivo (ONU-Hábitat, 2003; 2009). 
Al combinar la información de la EPH-Indec sobre la fuente de origen del agua y la localización, generamos una matriz de valores que representan niveles. En este sentido, cualquier situación que no brinde agua por cañería dentro de la vivienda, o que no sea de red pública o perforación con bomba a motor, requiere esfuerzo físico o pérdida de tiempo. Una combinación de ambas situaciones deficitarias o la sola ubicación fuera del terreno, representa un grave déficit (Tabla 3).

\section{3. Índice sobre disposición de excretas y saneamiento (IDE)}

La información estadística disponible está relacionada con tres subdimensiones que resultan indispensables para la seguridad sanitaria: 1) la localización del baño; 2) tipo de arrastre, y 3) el desagüe. La situación de déficit de saneamiento estaría dada con la presencia de: 1) localización del baño fuera del terreno; 2) tipo de arrastre letrina; o 3) desagüe tipo hoyo (Córdova, 1999; Rodríguez, 1999; Szalachman, 2000; ONU-Hábitat, 2009). Es decir, que la sola presencia de una de estas características implica un déficit de saneamiento.

Tomando como base estos criterios asignamos un puntaje a cada categoría de las mencionadas subdimensiones. Respecto a la localización del baño, si está dentro de la vivienda: 1 punto; fuera de la vivienda, pero dentro terreno: 0,75 puntos; fuera terreno: 0,20 puntos. Esto significa que es aceptable tener un baño dentro del terreno, pero un déficit tenerlo por fuera. En cuanto al tipo de arrastre: Inodoro con botón/mochila/cadena y arrastre de agua: 1 punto; inodoro sin botón/ cadena y con arrastre de agua (a balde): 0,80 puntos; letrina (sin arrastre de agua): 0,30 puntos.

Por otra parte, el desagüe es adecuado cuando se encuentra conectado al alcantarillado o cámara séptica (Szalachman, 2000; ONU-Hábitat, 2003). En este sentido se asignaron los siguientes puntajes: a red pública (cloaca): 1 punto; a cámara séptica: 0,90 puntos; solo a pozo ciego: 0,65 puntos; a hoyo/ excavación en la tierra: 0,20 puntos. La cámara séptica la consideramos un poco inferior al desagüe por red, debido a que existen diferentes tipos de métodos de construcción y calidad (Marińelarena, 2006), algunos de los cuales no son tan adecuados como la red cloacal. El pozo ciego no llega a ser aceptable en términos de higiene, pero sí supera el mínimo necesario. Mientras que el hoyo representa un déficit, tal como se mencionó con anterioridad. Por último, si la vivienda no tiene baño el puntaje es 0 (cero).

En la Tabla 4 están representados todos los posibles valores del índice de disposición de excretas y saneamiento, que surgen del producto de los mencionados valores.

\section{4. Índice de personas por dormitorio (IPD)}

Como mencionamos en el apartado 2, en esta dimensión consideramos solo la relación personas por cuarto y no la referida a la cantidad de hogares por vivienda. Las razones son tanto metodológicas como empíricas. Respecto a la primera,

Tabla 3

Índice de acceso al agua (IAA)

\begin{tabular}{cccc} 
Fuente & $\begin{array}{c}\text { Por cañería dentro } \\
\text { de la vivienda }\end{array}$ & $\begin{array}{c}\text { Fuera de la vivienda, pero } \\
\text { dentro del terreno }\end{array}$ & Fuera del terreno \\
\hline $\begin{array}{c}\text { Red pública (agua corriente) } 0 \\
\text { perforación con bomba a motor }\end{array}$ & 1 & 0.4 & 0.15 \\
\hline $\begin{array}{c}\text { Perforación con bomba manual u } \\
\text { otra fuente }\end{array}$ & 0.4 & 0.16 & 0.06 \\
\hline
\end{tabular}

Fuente: elaboración propia 
Tabla 4

Índice de disposición de excretas y saneamiento (IDE)

\begin{tabular}{|c|c|c|c|c|c|}
\hline \multirow{2}{*}{ Localización } & \multirow{2}{*}{ Tipo de arrastre } & \multicolumn{4}{|c|}{ Desagüe } \\
\hline & & Red & Cámara & Pozo ciego & Hoyo \\
\hline \multirow{3}{*}{ Dentro } & Inodoro c/botón & 1 & 0.9 & 0.65 & 0.2 \\
\hline & A balde & 0.8 & 0.72 & 0.52 & 0.16 \\
\hline & Letrina & $0,30^{* *}$ & $0,27^{\star \star}$ & 0,20 & $0,06^{\star *}$ \\
\hline \multirow{3}{*}{$\begin{array}{l}\text { Fuera vivienda, } \\
\text { dentro terreno }\end{array}$} & Inodoro c/botón & 0.75 & 0.68 & 0.49 & $0,15^{\star *}$ \\
\hline & A balde & 0.6 & 0.54 & 0.39 & 0.12 \\
\hline & Letrina & $0,23^{\star *}$ & 0,20 & $0,15^{\star \star}$ & 0.05 \\
\hline \multirow{3}{*}{ Fuera terreno } & Inodoro c/ botón & 0,20 & $0,18^{\star *}$ & $0,13^{\star *}$ & $0,04^{* *}$ \\
\hline & A balde & 0,16 & $0,14^{\star *}$ & 0.1 & $0,03^{* *}$ \\
\hline & Letrina & $0,06^{* *}$ & $0,05^{\star \star}$ & $0,04^{\star \star}$ & 0,01 \\
\hline
\end{tabular}

Fuente: elaboración propia

** No existe algún caso de estas combinaciones en el IV trimestre de 2014.

el indicador seleccionado permite medir niveles en los términos de la propuesta de medición del apartado 3, al poseer puntos de corte o umbrales en los valores de hacinamiento crítico y semicrítico. En cuanto a la segunda razón, la evidencia empírica da cuenta de la muy baja proporción de hogares que comparten vivienda en la muestra de la EPH-Indec, que podría ser consecuencia de los problemas de captación del relevamiento ${ }^{4}$. En consecuencia, el indicador seleccionado es el que mide la relación entre la cantidad de personas de cada hogar y los cuartos que tienen a su disposición.

Para definir los valores de referencia, tuvimos en cuenta tanto algunas cuestiones empíricas como también los umbrales de hacinamiento crítico y semicrítico de distintos países —en particular Argentina- (Lentini y Palero, 1997; ONU-Hábitat, 2009; Rodríguez, 1999; Szalachman, 2000). Debido a que el número de dormitorios es una variable acotada, la definición del umbral para el nivel de hacinamiento puede afectar con fuerza los resultados. En nuestra propuesta, un hogar constituido por un matrimonio y dos hijos que cuente con dos dormitorios, sería una situación adecuada — dos personas por dormitorio-. Si llega un tercer hijo a ese hogar y se cuenta con la misma cantidad de habitaciones, las condiciones pasarían a estar en el umbral de lo aceptable $-2,5$ personas- - Un cuarto hijo con la misma infraestructura ubicaría a esta familia en el umbral de lo mínimo - tres personas-. Más allá de este valor, aparece el hacinamiento en sus diversas formas — semicrítico y crítico- (Tabla 5).

Tabla 5

Índice de personas por dormitorio (IPD)

\begin{tabular}{|c|c|c|}
\hline $\begin{array}{l}\text { Intervalos de } \\
\text { personas por } \\
\text { dormitorio }\end{array}$ & Intervalos del IPD & $\begin{array}{l}\text { Grado de } \\
\text { bienestar }\end{array}$ \\
\hline 20 menos & 1 & Alto \\
\hline$(2 ; 2,5)$ & $(0,70 ; 1)$ & \multirow{2}{*}{ Medio-alto } \\
\hline 2.5 & 0.7 & \\
\hline$(2,5 ; 3)$ & $(0,40 ; 0,70)$ & \multirow{2}{*}{ Medio-bajo } \\
\hline 3 & 0.4 & \\
\hline$(3 ; 5)$ & $(0,15 ; 0,40)$ & \multirow{3}{*}{ Bajo } \\
\hline 5 & 0.15 & \\
\hline$(5 ; 10)$ & $(0 ; 0,15)$ & \\
\hline 100 más & 0 & Nulo \\
\hline
\end{tabular}

4 En el IV trimestre de 2014 tan solo un 1,6 \% de los hogares comparten vivienda. De ese número, un $40 \%$ ya es captado con el IPD con valores menores a 0,40 y $60 \%$ restante posee un $40 \%$ de los hogares con un solo integrante —unipersonales-. En definitiva, un $1 \%$ del total de hogares se encuentra con dos o más integrantes que comparten vivienda y no está incluido en el IPD con valor menor a 0,40. La baja proporción de hacinamiento por cohabitación puede ser consecuencia tanto del marco muestral de la EPH-Indec como también de ciertas limitaciones en los procedimientos de captación de los hogares que comparten una unidad de vivienda, tal como ha sucedido en los censos de población y vivienda (DGEC, 2013). En este sentido, su inclusión en el cálculo del IPD puede generar sesgos indeseados. 
- Propietario de la vivienda y el terreno. Es

El cálculo para los niveles intermedios se realizó con la fórmula de LST por tramos, lo que arrojó una relación funcional muy sensible a la incorporación de un nuevo habitante en el primer intervalo - hasta tres personas por cuarto- , con sensibilidad relativa entre tres y cinco personas por dormitorio, y poco sensible a partir de las cinco personas por dormitorio.

\section{5. Índice sobre seguridad jurídica en la tenencia (ISJT)}

Por lo general, a esta variable se la divide en dos categorías: tenencia regular y tenencia irregular (ONU-Hábitat, 2003; 2009). Sin embargo, basándonos en el CCC distinguimos entre los criterios de legalidad, posesión y tenencia. A partir de allí, reagrupamos las ocho categorías definidas en la EPH-Indec en tres grupos — posesión legal, tenencia legal y posesión ilegal—y luego asignamos el puntaje (Tabla 6).

Tabla 6

Índice sobre seguridad jurídica en la tenencia (ISJT)

Categoria Régimen de tenencia Puntaje

\begin{tabular}{|c|c|c|}
\hline \multirow{2}{*}{ 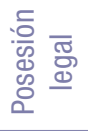 } & $\begin{array}{c}\text { Propietario de la vivienda } \\
\text { y del terreno }\end{array}$ & 1 \\
\hline & Está en sucesión & 0.85 \\
\hline \multirow{4}{*}{ 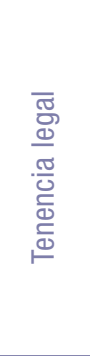 } & $\begin{array}{l}\text { Inquilino/arrendatario } \\
\text { de la vivienda }\end{array}$ & 0.7 \\
\hline & $\begin{array}{l}\text { Ocupante en relación } \\
\text { de dependencia }\end{array}$ & 0.5 \\
\hline & $\begin{array}{l}\text { Ocupante gratuito } \\
\text { (con permiso) }\end{array}$ & 0.5 \\
\hline & $\begin{array}{l}\text { Ocupante por pago de } \\
\text { impuestos/expensas }\end{array}$ & 0.5 \\
\hline \multirow{2}{*}{ 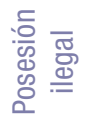 } & Propietario solo de la vivienda & 0.15 \\
\hline & $\begin{array}{l}\text { Ocupante de hecho } \\
\text { (sin permiso) }\end{array}$ & 0 \\
\hline
\end{tabular}

Fuente: elaboración propia

\subsubsection{Posesión legal}

aquel que tiene derecho a usar y disponer de la vivienda aunque esté pendiente de pago parcial. La propiedad es el dominio pleno de los conceptos posesión y tenencia, e incluye la mayor cantidad de facultades posibles que alguien pueda tener sobre una cosa, por ende, le asignamos 1 punto.

, En sucesión. Situación en que existe transmisión de los derechos activos y pasivos de una persona muerta a la persona que sobrevive -Artículo 3279 del CCC-. De acuerdo con el Artículo 3410 del CCC, una vivienda en sucesión se la considera como posesión. El puntaje asignado es de 0,85 para distinguirlo de la propiedad, debido a las posibles situaciones de conflicto familiar o la posible subdivisión.

\subsubsection{Tenencia legal}

Inquilino/arrendatario de la vivienda. Aquel que debe pagar para el uso de la vivienda un monto determinado por su propietario. Es la figura clásica de tenedor dado que tiene la cosa pero reconoce en otra persona la propiedad de la misma. Al ser una situación aceptable, el puntaje es 0,70 .

Ocupante gratuito (con permiso) y ocupante por pago de impuestos/expensas. En el primer caso, la persona usa la vivienda en calidad de préstamo, sin erogaciones, y en la segunda debe hacerse cargo de los gastos. Estas dos categorías se encuadran en la figura del contrato real de mutuo normado por el CCC, desde el Artículo 2240 hasta el 2254, y pueden dividirse en dos: regulares y precarias, dependiendo si se encuentra o no establecido un plazo de permanencia. Por lo tanto, las regulares tendrían un puntaje de 0,70 , mientras que las precarias de 0,30 . No obstante, dado que esta información no se encuentra disponible en la EPH-Indec, decidimos 
asignar un puntaje de 0,50, bajo la hipótesis que existe igual cantidad de situaciones regulares y precarias.

- Ocupante con relación de dependencia. Es el caso del hogar que recibe el uso de la vivienda como parte de pago por el trabajo de alguno o algunos de sus integrantes. Esta situación se encuadra en una relación jurídica diferente, es decir, en el marco de las relaciones laborales. Es decir, un trabajo permanente - por ejemplo, el encargado de un edificio con estabilidad laboral- sería una circunstancia adecuada con puntaje 0,70 . En cambio, un trabajador inestable — un sereno con finalización de contrato por tarea-, tendría un puntaje de 0,30 . De nuevo, dado que la información disponible no permite reconocer qué integrante del hogar es el que recibe como pago la vivienda, asignamos un puntaje de 0,50 basado en la misma hipótesis anterior.

\subsubsection{Posesión ilegal}

- Propietario solo de la vivienda. Cuando no tiene la posesión del terreno en que está la vivienda $y$, por lo tanto, el hecho posesorio no es legítimo. Puntaje 0,15.

- Ocupante de hecho (sin permiso). El hogar hace uso de la vivienda sin autorización de su propietario. Se incluyen en esta categoría todos los casos de usurpaciones de viviendas o edificios. Puntaje 0.

La Tabla 6 resume la información respecto al puntaje y categoría jurídica.

En el siguiente apartado, aplicamos esta propuesta con datos de Argentina para validar la versatilidad del conjunto de índices. Consideramos que el potencial de este tipo de mediciones excede el ejercicio propuesto, dado que al construir escalas con estándares internacionales, se podrían efectuar comparaciones entre países. La limitación está dada en el IPD y el ISJT, ya que poseen criterios específicos a normas o costumbres.

\section{Resultados del ejercicio de validación}

El análisis de resultados lo dividimos en dos partes. La primera corresponde al examen distributivo, donde calculamos los I-Med, I-Des y la pérdida de bienestar por la desigualdad en los 29 aglomerados urbanos que componen la muestra de la EPH-Indec en el IV trimestre de 2014. La segunda, al estudio de las correlaciones bivariadas mediante el uso del coeficiente de Pearson.

\subsection{Análisis de las medidas de distribución}

\subsection{1. Índice de calidad del techo-piso (ITP)}

El ITP-Med resultó mayor a 0,70 en todos los aglomerados (Tabla 7). No obstante, se presentan fuertes desigualdades en algunos territorios. En los mejor posicionados, casi todas las viviendas poseen techo y piso adecuados. En cambio, en los peor calificados la distribución presenta sesgos. Por ejemplo, en Gran San Juan (29) la población del primer cuartil posee como máximo un nivel de techo-piso de 0,42 , que significa un peso de cemento o ladrillo fijo y un techo de chapa de metal sin cubierta o chapa de fibrocemento/plástico sin cielorraso (Figura 1a).

Al calcular el ITP-Des, el valor disminuye en todos los aglomerados, al estar en mayor proporción en los peor ubicados (Tabla 8). Lo anterior se puede observar al estudiar la asociación entre el ITP-Med y el porcentaje de pérdida de bienestar por desigualdad, que arroja una correlación muy alta con un $\mathrm{R}^{2}$ de 0,916 (Figura 2a).

\subsection{2. Índice de acceso al agua (IAA)}


Tabla 7

Resultados de Ios I-Med. Aglomerados ordenados con base en la calificación de ICH-Med

\begin{tabular}{|c|c|c|c|c|c|c|c|}
\hline \multirow{2}{*}{ Categoría } & \multirow{2}{*}{ Aglomerado } & \multicolumn{6}{|c|}{ I-Med } \\
\hline & & ITP & IAA & IDE & IPD & ISJT & $\mathrm{ICH}$ \\
\hline 1 & Santa Rosa-Toay & 0.986 & 0.999 & 0.991 & 0.857 & 0.923 & 0.897 \\
\hline 2 & Mar del Plata & 0.965 & 0.992 & 0.975 & 0.876 & 0.882 & 0.890 \\
\hline 3 & Río Gallegos & 0.982 & 1.000 & 0.995 & 0.873 & 0.837 & 0.877 \\
\hline 4 & Ciudad Autónoma de Buenos Aires & 0.989 & 0.996 & 0.997 & 0.891 & 0.805 & 0.869 \\
\hline 5 & Bahía Blanca-Cerri & 0.961 & 0.993 & 0.956 & 0.854 & 0.855 & 0.858 \\
\hline 6 & La Rioja & 0.924 & 0.998 & 0.982 & 0.820 & 0.910 & 0.857 \\
\hline 7 & Ushuaia-Río Grande & 0.997 & 0.961 & 0.990 & 0.883 & 0.821 & 0.857 \\
\hline 8 & Gran Mendoza & 0.929 & 0.993 & 0.976 & 0.855 & 0.840 & 0.852 \\
\hline 9 & Río Cuarto & 0.930 & 0.989 & 0.954 & 0.853 & 0.839 & 0.851 \\
\hline 10 & Neuquén-Plottier & 0.958 & 0.997 & 0.979 & 0.815 & 0.886 & 0.851 \\
\hline 11 & San Luis-El Chorrillo & 0.957 & 0.998 & 0.978 & 0.782 & 0.907 & 0.845 \\
\hline 12 & Jujuy-Palpalá & 0.848 & 0.976 & 0.970 & 0.825 & 0.924 & 0.832 \\
\hline 13 & Gran La Plata & 0.916 & 0.989 & 0.895 & 0.849 & 0.863 & 0.831 \\
\hline 14 & Gran Paraná & 0.933 & 0.993 & 0.932 & 0.850 & 0.803 & 0.830 \\
\hline 15 & Gran Rosario & 0.931 & 0.974 & 0.907 & 0.825 & 0.851 & 0.816 \\
\hline 16 & Gran Catamarca & 0.921 & 0.966 & 0.931 & 0.769 & 0.927 & 0.814 \\
\hline 17 & Gran Santa Fe & 0.934 & 0.998 & 0.840 & 0.844 & 0.842 & 0.811 \\
\hline 18 & Gran Córdoba & 0.940 & 0.981 & 0.919 & 0.791 & 0.834 & 0.805 \\
\hline 19 & Total aglomerados & 0.900 & 0.970 & 0.905 & 0.807 & 0.843 & 0.800 \\
\hline 20 & Corrientes & 0.883 & 0.980 & 0.933 & 0.765 & 0.888 & 0.798 \\
\hline 21 & Comodoro Rivadavia-Rada Tilly & 0.971 & 0.985 & 0.953 & 0.783 & 0.801 & 0.795 \\
\hline 22 & Posadas & 0.877 & 0.952 & 0.861 & 0.813 & 0.826 & 0.792 \\
\hline 23 & Gran Resistencia & 0.899 & 0.976 & 0.928 & 0.763 & 0.858 & 0.786 \\
\hline 24 & Santiago del Estero-La Banda & 0.913 & 0.908 & 0.842 & 0.752 & 0.939 & 0.777 \\
\hline 25 & Salta & 0.823 & 0.951 & 0.921 & 0.793 & 0.850 & 0.772 \\
\hline 26 & Partidos del GBA & 0.867 & 0.957 & 0.862 & 0.774 & 0.836 & 0.764 \\
\hline 27 & Concordia & 0.847 & 0.968 & 0.927 & 0.756 & 0.807 & 0.759 \\
\hline 28 & Gran San Juan & 0.773 & 0.985 & 0.831 & 0.809 & 0.868 & 0.756 \\
\hline 29 & Formosa & 0.816 & 0.904 & 0.934 & 0.725 & 0.905 & 0.756 \\
\hline 30 & Gran Tucumán-Tafí Viejo & 0.819 & 0.933 & 0.859 & 0.781 & 0.811 & 0.747 \\
\hline
\end{tabular}

Fuente: elaboración propia con base en microdatos EPH-INDEC del IV trimestre de 2014

Tabla 8

Resultados de los I-Des. Aglomerados ordenados con base en la calificación de ICH-Des

\begin{tabular}{cccccccc} 
Categoría & Aglomerado & \multicolumn{4}{c}{ I-Des } \\
& & ITP & IAA & IDE & IPD & ISJT & ICH \\
\hline 1 & Santa Rosa-Toay & 0.978 & 0.999 & 0.989 & 0.793 & 0.904 & 0.870 \\
\hline 2 & Mar del Plata & 0.949 & 0.987 & 0.969 & 0.822 & 0.848 & 0.861 \\
\hline 3 & Río Gallegos & 0.973 & 1.000 & 0.992 & 0.813 & 0.802 & 0.847 \\
\hline 4 & Ciudad Autónoma de Buenos Aires & 0.985 & 0.994 & 0.996 & 0.837 & 0.752 & 0.836 \\
\hline 5 & La Rioja & 0.902 & 0.997 & 0.978 & 0.750 & 0.880 & 0.825 \\
\hline
\end{tabular}




\begin{tabular}{|c|c|c|c|c|c|c|c|}
\hline \multirow{2}{*}{ Categoría } & \multirow{2}{*}{ Aglomerado } & \multicolumn{6}{|c|}{ I-Des } \\
\hline & & ITP & IAA & IDE & IPD & ISJT & $\mathrm{ICH}$ \\
\hline 6 & Ushuaia-Río Grande & 0.996 & 0.934 & 0.987 & 0.828 & 0.764 & 0.820 \\
\hline 7 & Bahía Blanca-Cerri & 0.941 & 0.988 & 0.943 & 0.784 & 0.812 & 0.819 \\
\hline 8 & Gran Mendoza & 0.908 & 0.990 & 0.964 & 0.791 & 0.796 & 0.814 \\
\hline 9 & Neuquén-Plottier & 0.941 & 0.995 & 0.973 & 0.738 & 0.853 & 0.813 \\
\hline 10 & Río Cuarto & 0.910 & 0.981 & 0.933 & 0.786 & 0.799 & 0.811 \\
\hline 11 & San Luis-El Chorrillo & 0.940 & 0.997 & 0.972 & 0.702 & 0.879 & 0.808 \\
\hline 12 & Jujuy-Palpalá & 0.811 & 0.962 & 0.959 & 0.753 & 0.895 & 0.796 \\
\hline 13 & Gran Paraná & 0.906 & 0.988 & 0.907 & 0.787 & 0.741 & 0.789 \\
\hline 14 & Gran La Plata & 0.884 & 0.983 & 0.870 & 0.779 & 0.808 & 0.788 \\
\hline 15 & Gran Santa Fe & 0.908 & 0.996 & 0.815 & 0.779 & 0.787 & 0.775 \\
\hline 16 & Gran Rosario & 0.906 & 0.959 & 0.883 & 0.755 & 0.796 & 0.773 \\
\hline 17 & Gran Catamarca & 0.897 & 0.947 & 0.912 & 0.689 & 0.899 & 0.769 \\
\hline 18 & Gran Córdoba & 0.925 & 0.969 & 0.910 & 0.710 & 0.790 & 0.765 \\
\hline 19 & Total aglomerados & 0.867 & 0.953 & 0.882 & 0.731 & 0.788 & 0.755 \\
\hline 20 & Corrientes & 0.844 & 0.968 & 0.900 & 0.685 & 0.842 & 0.750 \\
\hline 21 & Comodoro Rivadavia-Rada Tilly & 0.960 & 0.976 & 0.933 & 0.704 & 0.732 & 0.747 \\
\hline 22 & Posadas & 0.841 & 0.922 & 0.819 & 0.740 & 0.756 & 0.741 \\
\hline 23 & Gran Resistencia & 0.865 & 0.963 & 0.912 & 0.679 & 0.793 & 0.740 \\
\hline 24 & Santiago del Estero-La Banda & 0.886 & 0.865 & 0.795 & 0.678 & 0.911 & 0.728 \\
\hline 25 & Salta & 0.777 & 0.922 & 0.887 & 0.713 & 0.789 & 0.721 \\
\hline 26 & Partidos del GBA & 0.828 & 0.933 & 0.836 & 0.695 & 0.776 & 0.717 \\
\hline 27 & Gran San Juan & 0.693 & 0.974 & 0.805 & 0.733 & 0.822 & 0.711 \\
\hline 28 & Concordia & 0.798 & 0.951 & 0.898 & 0.665 & 0.736 & 0.705 \\
\hline 29 & Formosa & 0.771 & 0.860 & 0.914 & 0.633 & 0.855 & 0.704 \\
\hline 30 & Gran Tucumán-Tafí Viejo & 0.769 & 0.899 & 0.822 & 0.702 & 0.739 & 0.699 \\
\hline
\end{tabular}

Fuente: elaboración propia con base en microdatos del EPH-INDEC del IV trimestre de 2014

El acceso al agua es alto en todos los aglomerados del país y oscila entre 0,90 y 1 . No obstante, en los niveles más bajos se encuentran los aglomerados de las regiones del noreste argentino (NEA) y del noroeste argentino (NOA) (Tabla 7). La distribución en algunos territorios evidencia la presencia de déficits importantes. En Formosa y Santiago del Estero, en el $5 \%$ más bajo de la distribución, hay personas con acceso al agua fuera del terreno - IAA de 0,15 - En algunos aglomerados del NEA y NOA, el decil más bajo tiene personas con un nivel de acceso al agua cercano al umbral deficitario $(0,40)$. Estas dispersiones no se llegan a apreciar en el diagrama de caja debido a que en todos los aglomerados, por lo menos un $75 \%$ de las personas tiene un IAA de 1 (Figura 1b).

Por otra parte, el IAA-Des es menor que el IAAMed en la mayor parte de los aglomerados. En este sentido, la pérdida de bienestar por desigualdad se ubica entre $0 \%$ —Río Gallegos-y 4,9\% - Formosa- (Tabla 9) y tiene una fuerte correlación respecto al acceso promedio al agua, dado que el $\mathrm{R}^{2}$ es de 0,982 (Figura 2b).

\subsection{3. Índice sobre disposición de excretas y saneamiento (IDE)}


Figura 1. Distribución de cada índice parcial en los aglomerados calificados $1^{\circ}, 10^{\circ}, 20^{\circ}$ y $29^{\circ}$
a) Índice de calidad del techo-piso (ITP)
b) Índice de acceso al agua (IAA)
c) Índice sobre disposición de excretas y saneamiento (IDE)
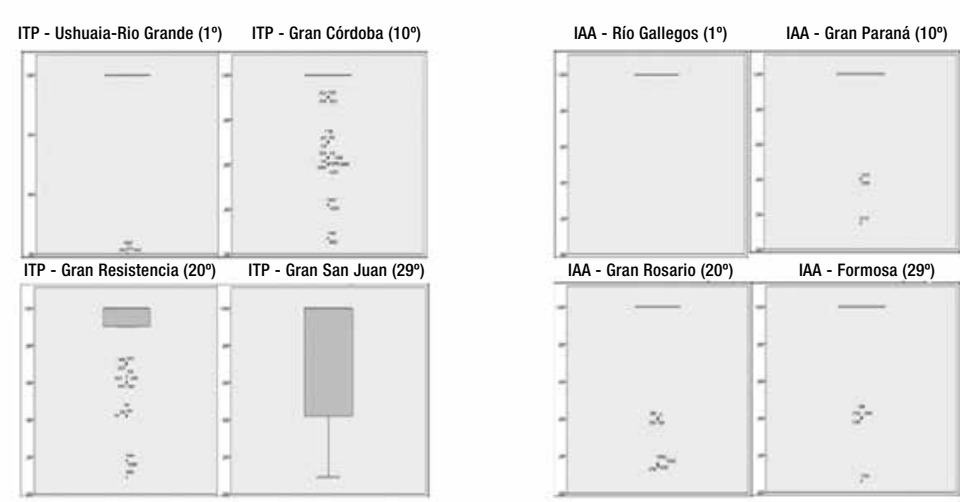

d) Índice de personas por dormitorio (IPD)

e) Índice sobre seguridad jurídica en la

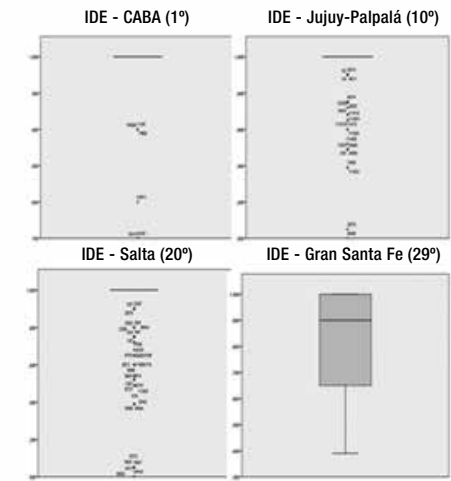
tenencia (ISJT)
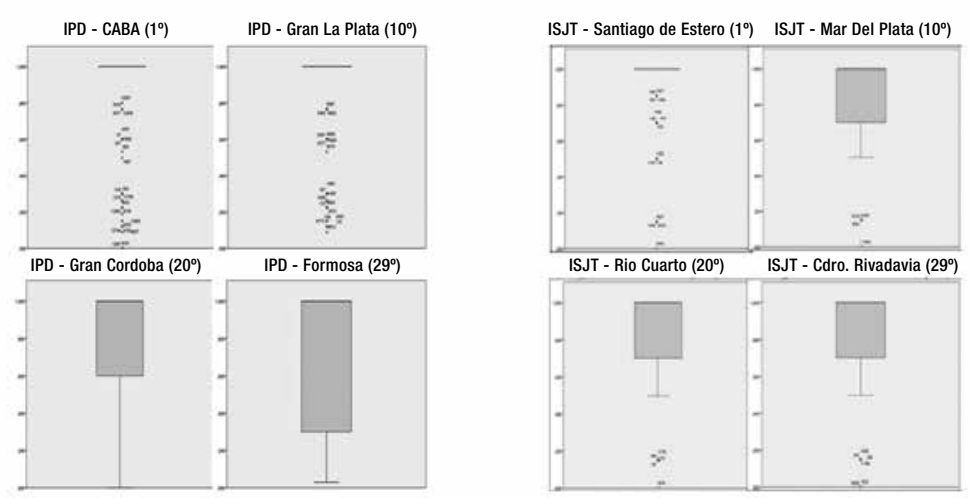

f) Índice de Condiciones Habitacionales (ICH)

Fuente: elaboración propia con base en microdatos EPH-Indec del IV trimestre de 2014

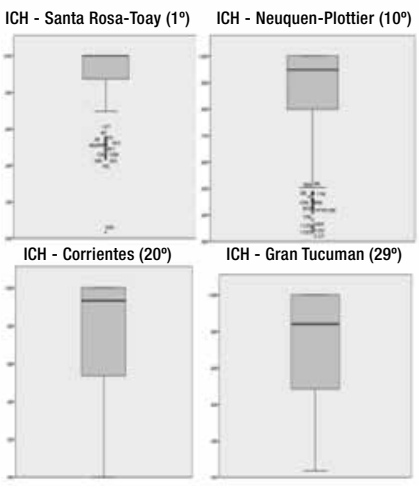

Figura 2. Relación de cada índice parcial con el porcentaje de pérdida de bienestar por desigualdad

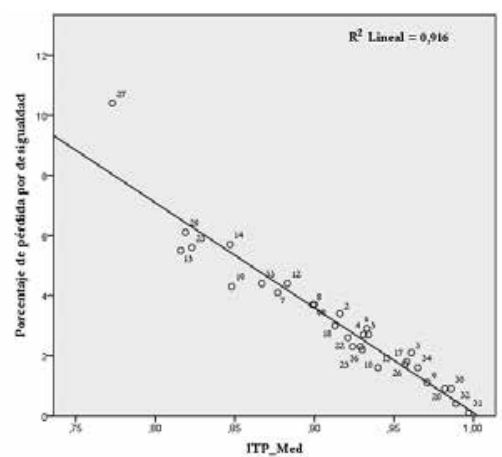

Índice de calidad del Techo-Piso

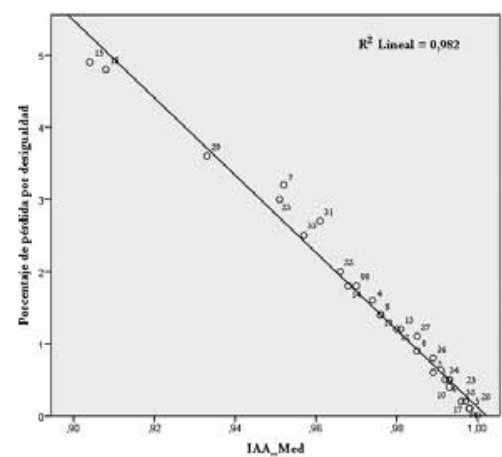

Índice de Acceso al Agua (IAA)

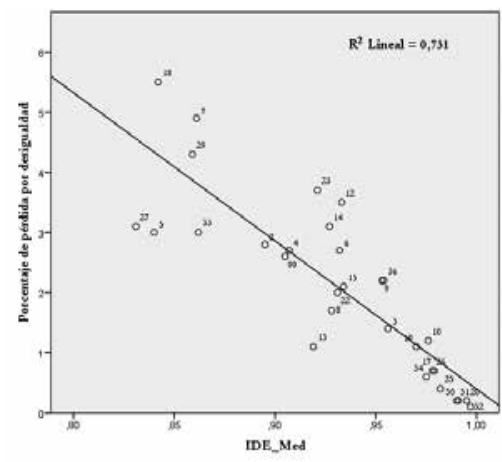

Índice sobre Disposición de Excertas y sanemaiento (IDE) 

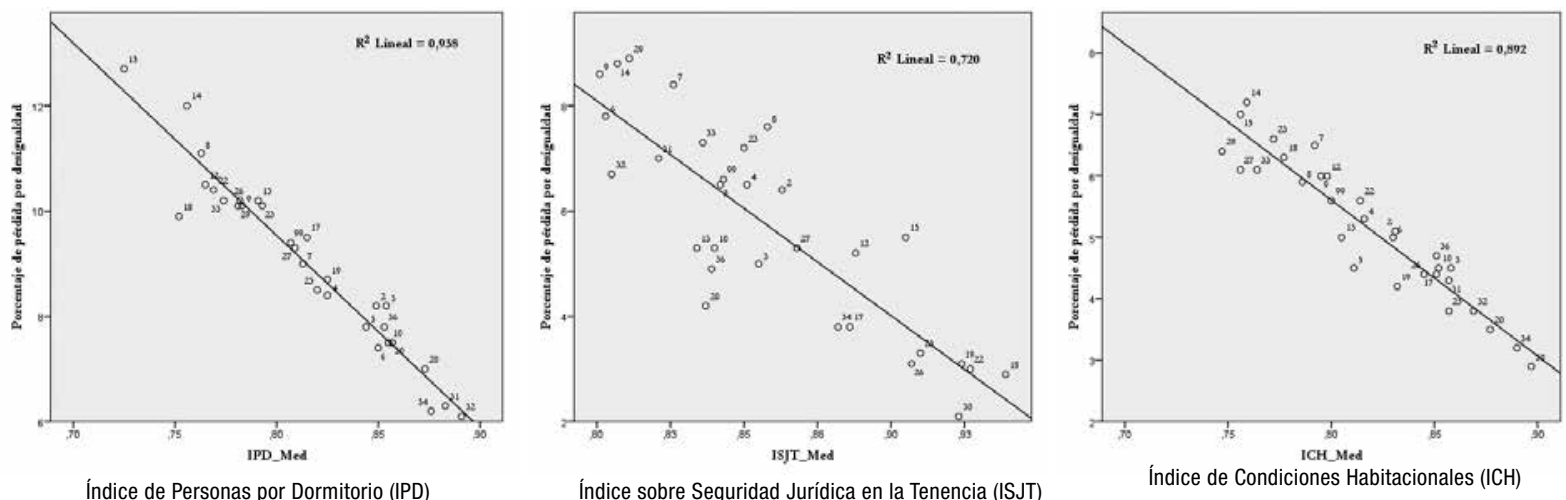

Fuente: elaboración propia con base en microdatos EPH-Indec del IV trimestre de 2014

(2) Gran La Plata; (3) Bahía Blanca-Cerri; (4) Gran Rosario; (5) Gran Santa Fe; (6) Gran Paraná; (7) Posadas; (8) Gran Resistencia; (9) Comodoro Rivadavia-Rada Tilly; (10) Gran Mendoza; (12) Corrientes; (13) Gran Córdoba; (14) Concordia; (15) Formosa; (17) Neuquén-Plottier; (18) Santiago del Estero-La Banda; (19) Jujuy-Palpalá; (20) Río Gallegos; (22) Gran Catamarca; (23) Salta; (25) La Rioja; (26) San Luis-El Chorrillo; (27) Gran San Juan; (29) Gran Tucumán-Tafí Viejo; (30) Santa Rosa-Toay; (31) Ushuaia-Río Grande; (32) CABA; (33) Partidos del GBA; (34) Mar del Plata; (36) Río Cuarto.

El IDE-Med arrojó valores entre 0,831 y 0,997. En las posiciones más bajas, las personas de los aglomerados de Santiago del Estero-La Banda, Gran Santa Fe y Gran San Juan, presentan situaciones promedio que representan un nivel de bienestar semejante a un baño dentro de la vivienda con arrastre a balde y desagüe a red. En el otro extremo, la Ciudad Autónoma de Buenos Aires (CABA), Río Gallegos y Santa RosaToay, en promedio se acercan a situaciones de bańo adecuado (Tabla 7).

Respecto a la distribución, a medida que se desciende en la calificación, comienza a aparecer una mayor dispersión de datos. Sin embargo, aun en la peor distribución, no se pierde el sesgo hacia los valores más altos. En los aglomerados peor ubicados se evidencian porcentajes significativos de personas bajo condiciones sanitarias deficitarias: en el percentil 5 hay población que como máximo tiene un inodoro con arrastre a balde dentro del terreno y con desagüe a pozo ciego - IDE de 0,39-. Esta situación se replica en el percentil 10, para el aglomerado de Santiago del Estero-La Banda. Y en el percentil 25, en los aglomerados de Gran Santa Fe y Gran San Juan el bańo se encuentra dentro de la vivienda y el arrastre es con botón pero con desagüe a pozo ciego $(0,65)$ (Figura 1c).

En virtud de ello, las repercusiones sobre el IDEDes son las esperadas, dado que el nivel de bienestar termina siendo peor en los aglomerados más desiguales (Tabla 8). Estas heterogeneidades entre aglomerados se traducen en los diferentes porcentajes de pérdidas de bienestar, que oscilan en un rango de $0,1 \%$ a 5,5 \% (Tabla 9). De nuevo, los aglomerados con peor distribución son los que poseen los niveles más bajos de IDE-Med, y viceversa, por lo tanto, se presenta una relación negativa $-R^{2}$ de $0,72-$ entre desigualdad y la disposición de excretas (Figura 2c).

\subsection{4. Índice de personas por dormitorio (IPD)}

Los aglomerados pertenecientes al NEA y NOA también presentan las peores situaciones, siendo Formosa $(0,725)$ y Santiago del Estero-La Banda $(0,752)$ los más perjudicados: en promedio conviven cerca de 2,5 personas por cuarto (Tabla 7). Respecto a la distribución, a medida que descendemos en la calificación se observa cómo se van dispersando los datos. En Formosa y Concordia se presentan situaciones de hacimiento en más de la cuarta parte de la población. En rigor, el 
percentil 5 incluye a personas que comparten un mismo dormitorio con cinco o más personas y el percentil 25 está conformado por aquellos que duermen en cuartos que comparten entre tres y cinco personas (Figura 1d).

El IPD-Des muestra una ordenación similar de aglomerados, pero ahora con siete de estos por debajo de 0,70 , diecinueve con valores entre $0,70 \mathrm{y}$ 0,80 , y tan solo cuatro con cifras superiores a 0,80 -CABA, Ushuaia-Río Grande, Mar del Plata y
Río Gallegos-. Es decir, que de acuerdo al índice ajustado por desigualdad, en la cuarta parte de los aglomerados la cantidad de personas por dormitorio es de 2,5 o más personas por cuarto, lo que representa un nivel medio-bajo (Tabla 8).

La pérdida de bienestar por desigualdad en la distribución es la más elevada y oscila entre un $6,1 \%$ y un $12,7 \%$ (Tabla 9 ), y esta tiene una relación inversa con el valor promedio, con un $\mathrm{R}^{2}$ de 0,938 (Figura 2d).

Tabla 9

Resultados de la pérdida de bienestar por desigualdad. Aglomerados ordenados con base en resultados del ICH

\begin{tabular}{|c|c|c|c|c|c|c|c|}
\hline \multirow{2}{*}{ Categoría } & \multirow{2}{*}{ Aglomerado } & \multicolumn{6}{|c|}{ Pérdida de bienestar por desigualdad } \\
\hline & & ITP & IAA & IDE & IPD & ISJT & $\mathrm{ICH}$ \\
\hline 1 & Santa Rosa-Toay & $0.9 \%$ & $0.0 \%$ & $0.2 \%$ & $7.5 \%$ & $2.1 \%$ & $2.9 \%$ \\
\hline 2 & Mar del Plata & $1.6 \%$ & $0.5 \%$ & $0.6 \%$ & $6.2 \%$ & $3.8 \%$ & $3.2 \%$ \\
\hline 3 & Río Gallegos & $0.9 \%$ & $0.0 \%$ & $0.2 \%$ & $7.0 \%$ & $4.2 \%$ & $3.5 \%$ \\
\hline 4 & La Rioja & $2.3 \%$ & $0.1 \%$ & $0.4 \%$ & $8.5 \%$ & $3.3 \%$ & $3.8 \%$ \\
\hline 5 & Ciudad Autónoma de Buenos Aires & $0.4 \%$ & $0.2 \%$ & $0.1 \%$ & $6.1 \%$ & $6.7 \%$ & $3.8 \%$ \\
\hline 6 & Jujuy-Palpalá & $4.3 \%$ & $1.4 \%$ & $1.1 \%$ & $8.7 \%$ & $3.1 \%$ & $4.2 \%$ \\
\hline 7 & Ushuaia-Río Grande & $0.1 \%$ & $2.7 \%$ & $0.2 \%$ & $6.3 \%$ & $7.0 \%$ & $4.3 \%$ \\
\hline 8 & San Luis-El Chorrillo & $1.7 \%$ & $0.1 \%$ & $0.7 \%$ & $10.2 \%$ & $3.1 \%$ & $4.4 \%$ \\
\hline 9 & Neuquén-Plottier & $1.8 \%$ & $0.2 \%$ & $0.7 \%$ & $9.5 \%$ & $3.8 \%$ & $4.4 \%$ \\
\hline 10 & Gran Santa Fe & $2.7 \%$ & $0.1 \%$ & $3.0 \%$ & $7.8 \%$ & $6.5 \%$ & $4.5 \%$ \\
\hline 11 & Bahía Blanca-Cerri & $2.1 \%$ & $0.5 \%$ & $1.4 \%$ & $8.2 \%$ & $5.0 \%$ & $4.5 \%$ \\
\hline 12 & Gran Mendoza & $2.3 \%$ & $0.4 \%$ & $1.2 \%$ & $7.5 \%$ & $5.3 \%$ & $4.5 \%$ \\
\hline 13 & Río Cuarto & $2.2 \%$ & $0.8 \%$ & $2.2 \%$ & $7.8 \%$ & $4.9 \%$ & $4.7 \%$ \\
\hline 14 & Gran Córdoba & $1.6 \%$ & $1.2 \%$ & $1.1 \%$ & $10.2 \%$ & $5.3 \%$ & $5.0 \%$ \\
\hline 15 & Gran Paraná & $2.9 \%$ & $0.5 \%$ & $2.7 \%$ & $7.4 \%$ & $7.8 \%$ & $5.0 \%$ \\
\hline 16 & Gran La Plata & $3.4 \%$ & $0.6 \%$ & $2.8 \%$ & $8.2 \%$ & $6.4 \%$ & $5.1 \%$ \\
\hline 17 & Gran Rosario & $2.7 \%$ & $1.6 \%$ & $2.7 \%$ & $8.4 \%$ & $6.5 \%$ & $5.3 \%$ \\
\hline 18 & Total aglomerados & $3.7 \%$ & $1.8 \%$ & $2.6 \%$ & $9.4 \%$ & $6.6 \%$ & $5.6 \%$ \\
\hline 19 & Gran Catamarca & $2.6 \%$ & $2.0 \%$ & $2.0 \%$ & $10.4 \%$ & $3.0 \%$ & $5.6 \%$ \\
\hline 20 & Gran Resistencia & $3.7 \%$ & $1.4 \%$ & $1.7 \%$ & $11.1 \%$ & $7.6 \%$ & $5.9 \%$ \\
\hline 21 & Corrientes & $4.4 \%$ & $1.2 \%$ & $3.5 \%$ & $10.5 \%$ & $5.2 \%$ & $6.0 \%$ \\
\hline 22 & Comodoro Rivadavia-Rada Tilly & $1.1 \%$ & $0.9 \%$ & $2.2 \%$ & $10.1 \%$ & $8.6 \%$ & $6.0 \%$ \\
\hline 23 & Gran San Juan & $10.4 \%$ & $1.1 \%$ & $3.1 \%$ & $9.3 \%$ & $5.3 \%$ & $6.1 \%$ \\
\hline 24 & Partidos del GBA & $4.4 \%$ & $2.5 \%$ & $3.0 \%$ & $10.2 \%$ & $7.3 \%$ & $6.1 \%$ \\
\hline 25 & Santiago del Estero-La Banda & $3.0 \%$ & $4.8 \%$ & $5.5 \%$ & $9.9 \%$ & $2.9 \%$ & $6.3 \%$ \\
\hline 26 & Gran Tucumán-Tafí Viejo & $6.1 \%$ & $3.6 \%$ & $4.3 \%$ & $10.1 \%$ & $8.9 \%$ & $6.4 \%$ \\
\hline 27 & Posadas & $4.1 \%$ & $3.2 \%$ & $4.9 \%$ & $9.0 \%$ & $8.4 \%$ & $6.5 \%$ \\
\hline 28 & Salta & $5.6 \%$ & $3.0 \%$ & $3.7 \%$ & $10.1 \%$ & $7.2 \%$ & $6.6 \%$ \\
\hline 29 & Formosa & $5.5 \%$ & $4.9 \%$ & $2.1 \%$ & $12.7 \%$ & $5.5 \%$ & $7.0 \%$ \\
\hline 30 & Concordia & $5.7 \%$ & $1.8 \%$ & $3.1 \%$ & $12.0 \%$ & $8.8 \%$ & $7.2 \%$ \\
\hline
\end{tabular}




\subsection{5. Índice sobre seguridad jurídica en la tenencia (ISJT)}

El ISJT-Med arrojó valores entre 0,801 y 0,939 (Tabla 7). No obstante, en seis aglomerados hay una proporción significativa de inseguridad en la tenencia, dado que en el $10 \%$ más bajo de la distribución hay personas que habitan en una vivienda donde ninguno de los habitantes es propietario del terreno - ISJT $=0,15-$. En veinte aglomerados, el $25 \%$ más bajo es inquilino. Tan solo en siete aglomerados el primer cuartil presenta propietarios de vivienda (Figura 1e).

El valor del ISJT-Des es menor que el ISJT-Med y es, en proporción, más bajo en los aglomerados peores ubicados. Las pérdidas de bienestar por desigualdad oscilan entre un 2,1\% y un 8,9 \% (Tabla 9). Estos valores se relacionan de forma negativa con los niveles de seguridad jurídica obtenidos por el ISJT-Med, con un $\mathrm{R}^{2}$ alto de 0,720 (Figura 2e).

\subsection{6. Índice de condiciones habitacionales (ICH)}

El ICH-Med estratifica los territorios en tres grupos bien definidos. Por un lado, un conjunto de once territorios con condiciones inferiores a 0,80 , que está integrado por todos los aglomerados del NEA y tres del NOA: Comodoro Rivadavia, Partidos del GBA, Concordia y Gran San Juan. Lo preocupante es que en el percentil 25 de estos territorios, perduran situaciones inferiores al nivel 0,40 , lo que significa que la cuarta parte de la población habita en hogares con más de un déficit habitacional.

Luego, un grupo de ocho aglomerados posee niveles entre 0,80 y 0,85 , donde las situaciones menores a 0,40 se dan en el $5 \%$ más bajo de la distribución. En los niveles más altos - entre 0,85 y 0,90 - se ubican diez aglomerados pertenecientes principalmente a las regiones pampeana, patagónica y CABA, y también de NOA y Cuyo (Tabla 7 y Figura 1f).
El ICH-Des resulta entre un 2,9 \% y un 7,2 \% más bajo que el ICH-Med, con lo cual, según esta medida, son dieciocho los aglomerados que poseen un nivel menor a 0,80. Gran Tucumán-Tafí Viejo es el único que se encuentra en el umbral de las condiciones aceptables, con un valor de 0,699 (Tablas 8 y 9). Los anteriores resultados ya marcan la pauta de la evidente relación negativa existente entre desigualdad y nivel promedio de las condiciones habitacionales, con un $R^{2}$ de 0,892 (Figura 2f).

Una vez analizado el promedio y la distribución de las condiciones habitacionales, resulta pertinente conocer las correlaciones bivariadas entre los índices que lo componen.

\subsection{Análisis de las correlaciones bivariadas del ICH}

El estudio de las correlaciones lo dividimos en dos partes. La primera, utilizando los datos individuales, es decir, respecto a la situación de cada una de las personas, lo que permite analizar la posible relación entre el grado de alcance de cada uno de los componentes habitacionales del hogar de forma personal. La segunda, con datos agregados, para conocer la relación entre los indicadores calculados por aglomerado.

\subsubsection{Correlaciones con datos individuales}

La relación lineal entre los distintos componentes es positiva, significativa a un $1 \%$, aunque moderada y baja. Los valores más altos, cercanos a 0,5 , corresponden a IDE con ITP y con IAA. Esto significa que algunas de las situaciones individuales de disposición de excretas están relacionadas con determinadas situaciones de techo-piso y acceso al agua, habiendo una moderada asociación entre la variabilidad en un índice respecto al cambio en otro. El resto de los índices presenta una asociación baja, menor a $0,4 \mathrm{y}$ mayor a 0,1 . 
En cambio, como es predecible, la relación de cada componente con la medida parcial del ICH presenta valores más elevados: con respecto al IPD es de 0,726 y con el ISJT es de 0,615. Es decir, la cantidad de personas por dormitorio en el hogar y la seguridad jurídica de la vivienda que habita una persona son las características más asociadas al nivel de las condiciones habitacionales. En cambio, el ITP y el IDE poseen una correlación moderada de entre 0,521 y 0,556 ; el IAA, una correlación baja de 0,380 (Tabla 10).

\subsubsection{Correlaciones con datos agregados}

Cuando analizamos estos resultados, las correlaciones son más altas debido a que la heterogeneidad de situaciones es menor que cuando lo hacemos con los resultados individuales.

Respecto a las asociaciones entre índices parciales, el ISJT-Med es el único que no se encuentra asociado de manera lineal y directa con el resto, con correlaciones no significativas tanto a $1 \%$ como a $5 \%$. Es decir, que la seguridad jurídica en la tenencia da cuenta de aspectos muy distintos a los que muestran el resto de los indicadores. En cambio, las correlaciones entre ITP, IAA, IDE e IPD son entre bajas y moderadas, con valores que oscilan entre 0,439 y 0,636 . Esto significa que algunos de los aglomerados que poseen un nivel promedio alto de techo-piso, también tienen el mismo nivel promedio de acceso al agua, disposición de excretas, y personas por dormitorio, pero no el mismo nivel de seguridad jurídica en la tenencia. En cuanto a las correlaciones entre el ICH y cada uno de sus componentes, como es de esperar, los valores son de moderados a altos para ITP, IAA, IPD e IDE. Mientras que con ISJT la asociación es muy baja y no significativa (Tabla 11).

En cuanto a las estimaciones ajustadas por desigualdad, el ISJT-Des tampoco se encuentra asociado con ningún índice parcial y su correlación no es significativa. En cambio, el IDE-Des incrementa la correlación con respecto a los otros

Tabla 10

Correlación de Pearson entre el ICH y sus componentes. Datos individuales

\begin{tabular}{ccccccc} 
Índice & ITP & IAA & IDE & IPD & ISJT & ICH \\
ITP & 1 & & & & \\
\hline IAA & $0,365^{\star *}$ & 1 & & & & \\
\hline IDE & $0,518^{* *}$ & $0,503^{\star *}$ & 1 & & & \\
\hline IPD & $0,300^{* *}$ & $0,181^{* *}$ & $0,264^{* *}$ & 1 & & \\
\hline ISJT & $0,170^{* *}$ & $0,123^{* *}$ & $0,148^{* *}$ & $0,191^{* *}$ & 1 & 1 \\
\hline ICH & $0,556^{* *}$ & $0,380^{* *}$ & $0,521^{* *}$ & $0,726^{* *}$ & $0,615^{* *}$ & \\
\hline
\end{tabular}

Fuente: elaboración propia

** La correlación es significativa para 0,01 (2 colas).

Tabla 11

Correlación de Pearson entre los índices promedio (I-Med)

\begin{tabular}{ccccccc} 
Índice & ITP-Med & IAA-Med & IDE-Med & IPD-Med & ISJT-Med & ICH-Med \\
ITP-Med & 1 & & & & \\
\hline IAA-Med & $0,552^{* *}$ & 1 & & & & \\
\hline IDE-Med & $0,613^{* *}$ & $0,483^{* *}$ & 1 & & & \\
\hline IPD-Med & $0,602^{* *}$ & $0,636^{* *}$ & $0,439^{*}$ & 1 & & \\
\hline ISJT-Med & -0.067 & 0.14 & 0.096 & -0.266 & 1 & 1 \\
\hline ICH-Med & $0,816^{* *}$ & $0,690^{* *}$ & $0,761^{* *}$ & $0,801^{* *}$ & 0.153 &
\end{tabular}

Fuente: elaboración propia

** La correlación es significativa para 0,01 (2 colas). * La correlación es significativa para 0,05 (2 colas). 
índices parciales y el IPD-Des con respecto al ITP-Des. El resto disminuyen de forma leve la correlación. La explicación puede encontrarse en el siguiente argumento. Tal como demostramos en los apartados anteriores, el IDE-Med y el ISJT-Med poseen la menor asociación lineal entre desigualdad y bienestar, ya que presentan un $\mathrm{R}^{2}$ de 0,731 y 0,720 cada uno. En este sentido, inferimos que una vez que ajustamos los índices por desigualdad y le incorporamos el efecto de la varianza, sus ordenaciones quedan más similares a las del resto de los índices. Es decir, que generamos medidas que se asemejan en términos de variabilidad, dado que ahora se presenta una mayor proporción de varianza de uno de ellos asociada a otro de los índices (Tabla 12).

\section{Conclusiones}

Como se pudo constatar, con el ejercicio de aplicación para la Argentina, la propuesta de medición de las condiciones habitacionales permite transformar variables de distinta naturaleza en índices equiparables que representan niveles acordados por medio de un consenso intersubjetivo. De esta manera, se logra combinar intervalos con niveles ordinales que poseen una interpretación conceptual, donde los valores de referencia delimitan el déficit $(0,40)$ y la situación aceptable $(0,70)$. Asimismo, al incluir a todas las situaciones habitacionales en el cálculo, evita el riesgo de dejar afuera de las estimaciones a aquellos casos cercanos a los umbrales.
El alto grado de subjetividad, que permite la participación de especialistas en cada disciplina, es el ingrediente principal que lo diferencia de otro tipo de estimaciones. Ello no resulta en perjuicio de la medición, sino todo lo contrario, ya que permite despojarse del isomorfismo que caracteriza a los métodos más duros, que parten de forma implícita de una correspondencia entre la estructura lógico-matemática y la estructura de la realidad.

Los resultados obtenidos dan cuenta tanto de la desigualdad entre territorios, como también en estos. Respecto a lo interterritorial, si bien para el total de aglomerados el nivel de condiciones habitacionales promedio es medio-alto - $\mathrm{ICH}$ Med de 0,800-, la distancia entre los extremos de la calificación es de 0,150 , con valores desde 0,897 hasta 0,747 . Esta brecha se aumenta a 0,171 al corregir la medición por desigualdad - ICH-Des—, ubicando a Gran Tucumán-Tafí Viejo por debajo del umbral de condiciones promedio aceptables, con un valor de 0,699. En cuanto a la desigualdad intraterritorios, los porcentajes de pérdida de bienestar como consecuencia de la desigualdad, oscilan entre un 2,9\% y un $7,2 \%$, presentándose situaciones críticas en once aglomerados, dado que en el percentil 25 de estos territorios, perduran situaciones con más de un déficit habitacional.

Estas interpretaciones, que si bien se corresponden con la metodología propuesta, dependerán

Tabla 12. Correlación de Pearson entre los índices corregidos por desigualdad (I-Des)

\begin{tabular}{ccccccc} 
Índice & ITP-Des & IAA-Des & IDE-Des & IPD-Des & ISJT-Des & ICH-Des \\
\hline ITP-Des & 1 & & & & \\
\hline IAA-Des & $0,530^{* *}$ & 1 & & & \\
\hline IDE-Des & $0,650^{* *}$ & $0,534^{* *}$ & 1 & & \\
\hline IPD-Des & $0,604^{* *}$ & $0,604^{* *}$ & $0,462^{*}$ & 1 & 1 & 1 \\
\hline ISJT-Des & 0.049 & -0.007 & 0.202 & -0.134 & 0.289 & 1 \\
\hline ICH-Des & $0,804^{* *}$ & $0,706^{* *}$ & $0,778^{* *}$ & $0,810^{* *}$ & \\
\hline
\end{tabular}

Fuente: elaboración propia

** La correlación es significativa para 0,01 (2 colas). * La correlación es significativa para 0,05 (2 colas). 
de las precisiones metodológicas de la fuente de datos secundarios, es decir, la EPH-Indec.

Por otra parte, frente a las mencionadas ventajas de interpretación de resultados en una escala valorativa de cero a uno, hay que tener presente que, para traducir ese valor a un plano real de características habitacionales, el procedimiento es consistente pero no intuitivo. En el caso de los índices parciales resulta imprescindible la lectura de las tablas de equivalencias correspondientes. En cuanto al ICH, cuando el valor es menor a 0,40 solo se puede asegurar que se presenta más de una dimensión con déficit, pero no la cantidad exacta. No obstante, esto último no se encuentra en el objetivo de la propuesta, sino que corresponde a otro tipo de mediciones.

En este sentido, la medición de los valores promedio y de la distribución resulta de gran utilidad para evaluar y sugerir posibles cursos de acción de políticas públicas. La detección y corrección de las desigualdades habitacionales redundaría en mejores condiciones de salud y reduciría las problemáticas de pobreza estructural.

Por último, la propuesta presentada tiene la potencialidad de ser una herramienta para la comparación del nivel de las condiciones habitacionales entre países. Sin embargo, ello exige un desafío importante respecto a la definición de los umbrales, en el sentido que en el afán de obtener una medida comparable no se dejen de lado las características que son propias a cada cultura o Nación.

\section{Bibliografía}

Bostwick, G. J. y. Kyte, N. S. (2005). Measurement Social Work: Research and Evaluation Quantitative and Qualitative Approach. 7a. ed. Nueva York: Oxford University Press.
Cecchini, S. (2005). Propuesta para un compendio latinoamericano de indicadores sociales. Recuperado de http://www.cepal.org/deype/publicaciones/xml/0/27910/LCL2471e.pdf

Córdova, P. (1999). Características de la vivienda y del hogar. En Cepal (ed.), América Latina: aspectos conceptuales de los censos del 2000 (pp. 259274). Santiago: Cepal.

Dirección General de Estadísticas y Censos [DGEC]. (2013). Calidad de ocupación de las viviendas en la ciudad de Buenos Aires 2010. Buenos Aires. Autor.

Drewnowski, J. y Scott, W. (1966). The Level of Living Index. Ginebra: UNRISD.

Doyal, L. y Gough, I. (1994). Teoría de las necesidades humanas. Barcelona: Icaria.

García-Huidobro, G. (2005). Compendio de indicadores sociales. Santiago: Naciones Unidas.

Instituto Nacional de Estadísticas y Censos [Indec]. (2003). Calidad de los materiales de la vivienda, Calmat. Buenos Aires: Autor.

Instituto Argentino de Normalización [IRAM]. (1996). Norma IRAM 11603. Acondicionamiento térmico de edificios (CNA 5640). Recuperado de https://es.scribd.com/doc/58167249/ IRAM-11603

Lentini, M. y Palero, D. (agosto, 1997). El hacinamiento: la dimensión no visible del déficit habitacional. Boletín del Instituto de la Vivienda, 12(31), 23-32.

Madariaga, H. (2003). Propuesta de indice de necesidades básicas insatisfechas y su aplicación empleando sistemas de información geográfica. Tafí del Valle: AEPA. 
Mariñelarena, A. (2006). Manual de autoconstrucción de un sistema de tratamiento de aguas residuales domiciliarias. La Plata: Freplata.

Programa de Naciones Unidas para el Desarrollo [PNUD]. (2010). Informe sobre desarrollo humano 2010. Madrid: Mundiprensa Libros.

Ramírez, J. (2002). El juicio de desalojo. Buenos Aires: Nova Tesis.

Rodríguez, J. (1999). Información censal relevante para la medición del déficit habitacional. En Cepal (ed.), América Latina: aspectos conceptuales de los censos del 2000 (pp. 205-257). Santiago: Cepal.

Salvia, A. (2004). La deuda social argentina en el espacio del desarrollo. En A. Salvia y F. Tami (coords.), Barómetro de la deuda social argentina. Las grandes desigualdades. Buenos Aires: UCA.
Sen, A. (2000). Desarrollo y libertad. Barcelona: Planeta.

Spicker, P., Álvarez, S. y Gordon, D. (2009). Pobreza: un glosario internacional. Buenos Aires: Clacso.

Szalachman, R. (2000). Perfil de déficity politicas de vivienda de interés social: situación de algunos paises de la región en los noventa. Santiago: Cepal.

ONU-Hábitat. (2003). Guía para el monitoreo de la meta 11. Recuperado de http://onuhabitat.org/index.php?option=com_docman\&task=doc_download\&gid=146\&Itemid =71

ONU-Hábitat. (2009). Urban Indicators Guidelines. Recuperado de http://unhabitat.org/ urban-indicators-guidelines-monitoring-the-habitat-agenda-and-the-millennium-development-goals/ 